\title{
A NOVEL SPACE-VECTOR ALGORITHM FOR MULTILEVEL CONVERTERS BASED ON GEOMETRICAL CONSIDERATIONS USING A NEW SEQUENCE CONTROL TECHNIQUE
}

\author{
M. ÁNGELES MARTÍN PRATS, JOSE IGNACIO LEÓN GALVÁN, \\ RAMÓN PORTILLO GUISADO, JUAN MANUEL CARRASCO SOLÍS \\ and LEOPOLDO GARCIA FRANQUELO \\ Electronic Engineering Department, University of Seville, \\ Camino de los descubrimientos $s / n, 41092$ Sevilla, Spain
}

\begin{abstract}
This paper presents a fast and simple space vector modulation algorithm for voltage source multilevel converters for calculating the switching times and the space vectors using simple geometrical considerations. This method provides the nearest switching vectors sequence to the reference vector and calculates the on-state durations of the respective switching state vectors without involving trigonometric functions, look-up tables or coordinate system transformations which increase the computational load corresponding to the modulation of a multilevel converter. The low computational cost of the proposed method is always the same and it is independent of the number of levels of the converter. In addition, a new switching sequence control technique is presented for reducing the ripple of the DC-link voltage approximately in $66 \%$.
\end{abstract}

Keywords: Multilevel converter; space vector modulation; switching sequence; voltage ripple; step-by-step control algorithm.

\section{Introduction}

.

;

Although the advantages of multilevel converters were known since Akira Nabae proposed the Neutral Point Clamped (NPC) inverter topology in $1981,{ }^{1-3}$ its implementation was limited due to the complexity of the switching control. Recently, multilevel voltage source inverters have been used in medium and high power applications. They are capable of increasing the output voltage magnitude and reducing the output voltage and current harmonic content, the switching frequency and the voltage supported by each power semiconductor. Multilevel inverters permit to use the double voltage under the same type of switches. By synthesising the AC output voltage from several levels of voltages, staircase waveforms are produced and a sinusoidal waveform with low harmonic distortion is obtained. Multilevel converter enables the ac voltage to be increased without a transformer. The cancellation of low frequency harmonics from the ac voltages at the different levels means that the size of the ac inductances can be reduced. Due to these attractive characteristics, several control algorithms of multilevel converter have been recently proposed. ${ }^{4-8}$ The carrier-based PWM methods ${ }^{4-6}$ highly increase the algorithm complexity and 
the computational load with the number of levels of the multilevel converter, and the proposed space vector modulation algorithms involve trigonometric function calculations $^{8}$ or look-up tables or memories. ${ }^{7}$

As far as the authors know, the first space vector modulation for three-level and multilevel inverters for calculating the nearest switching vectors sequence to the reference vector and the on-state durations of the respective switching state vectors without trigonometric function calculations, look-up tables or coordinate system transformations was presented in Refs. 9 and 10. The results obtained using this iterative algorithm ${ }^{9,10}$ are improved using the fast modulation algorithm based on geometrical considerations proposed in this paper where a new switching sequence control technique is included for improving the performance of the converter. The proposed method can be applied to cascade, ${ }^{11}$ flying capacitor ${ }^{12}$ and $\mathrm{NPC}^{13}$ topologies.

\section{Algorithm Explanation}

\subsection{Introduction to the method}

Three-phase quantities are usually transformed into phasor representation for simplifying the calculations. Three vectors $\mathbf{v}_{1}, \mathbf{v}_{2}$ and $\mathbf{v}_{3}$ are used to approximate the desired voltage vector $\mathbf{v}^{*}$ in polar coordinates in a control cycle $T_{m}$. The modulation law requires the actual voltage vector $\mathbf{v}$ to equal its reference value $\mathbf{v}^{*}$ where $\mathbf{v}^{*}$ is represented in the stationary reference frame

$$
v=v^{*}=E_{a}+E_{b} e^{j 2 \pi / 3}+E_{c} e^{j 4 \pi / 3}=\operatorname{Re}\left\{v^{*}\right\}+j \operatorname{Img}\left\{v^{*}\right\} .
$$

During each modulation sub-cycle of duration $T_{m}$, a switching sequence is generated. It is composed of three switching state vectors $\mathbf{v}_{1}\left(t_{1}\right), \mathbf{v}_{2}\left(t_{2}\right)$ and $\mathbf{v}_{3}\left(t_{3}\right)$, where $t_{1}, t_{2}$ and $t_{3}$ are the on-state durations of the active switching state vectors. The three vectors nearest to the reference vector must be identified.

The input to the modulation algorithm of the multilevel converter is the normalized reference voltage vector $\mathbf{v}_{n}^{*}$. The states space normalization permits the developed algorithm to be independent of the DC-link voltage and the number of levels of voltages of the converter. ${ }^{9,10,14}$ In Fig. 1, the states space of a four-level converter in the complex plane $d-q$ is shown, where the coordinates of each of the vertex represent the levels of the DC-link voltage necessary to connect to each phase of the inverter to obtain the corresponding state. They take entire values between 0 and $n-1$, where $n$ is the number of the level of the multilevel converter.

The voltage vector $\mathbf{v}^{*}$ is transformed into $\mathbf{v}_{\text {flat }}^{*}$. This transformation consists of scaling imaginary part multiplying it by $1 / \sqrt{3} .9,10,14$ The transformation of $\mathbf{v}^{*}$ into $\mathbf{v}_{\text {flat }}^{*}$ allows us to use simple on-line computations of the states and switching times. ${ }^{9,10,14}$ The hexagon where the state vectors are represented is flattened and the three zones shown in Fig. 2 are found in the complex plane $d-q$.

In Fig. 3, the regular hexagon defined by the switching state vectors before and after the transformation in the complex plane is presented. ${ }^{10}$ The computations 


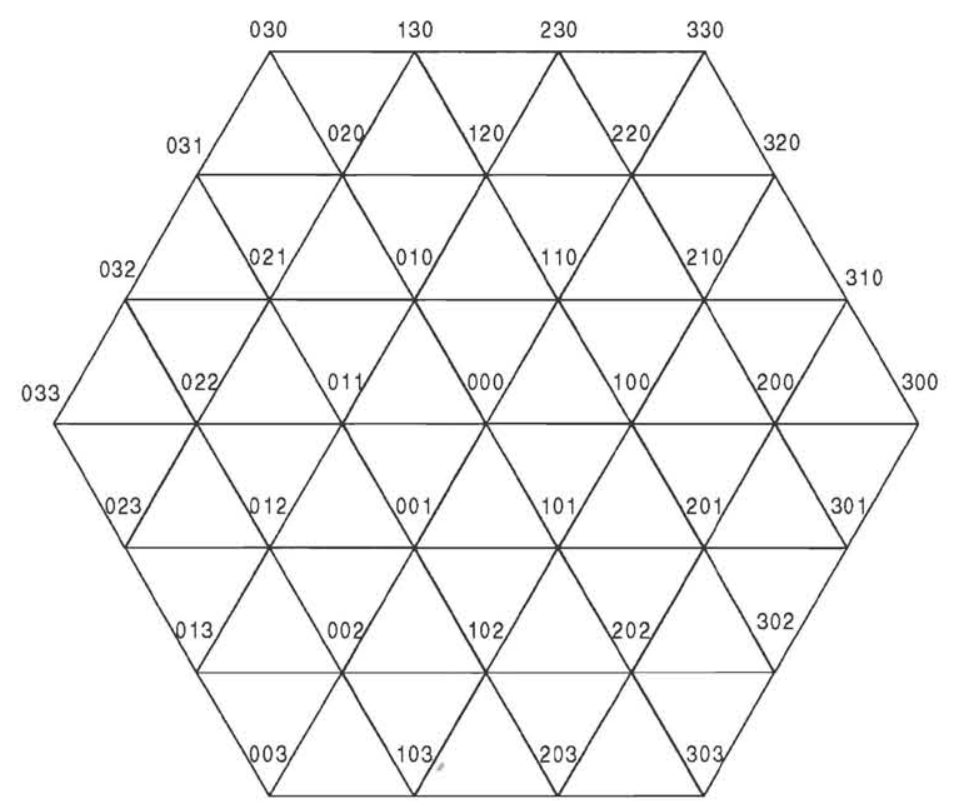

Fig. 1. Normalized states space of a four-level converter in the complex plane $d-q$.

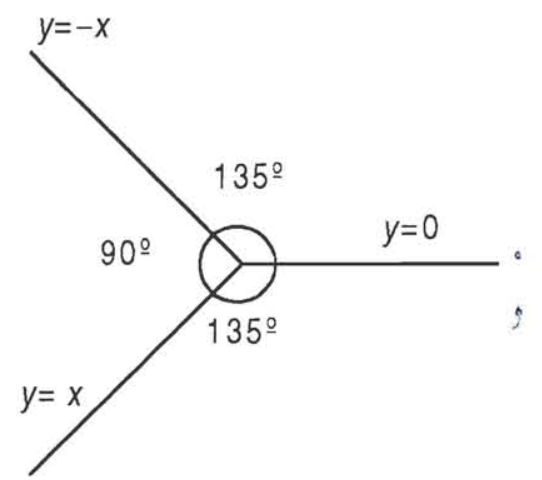

Fig. 2. Equations of the straight lines which divide the flattened complex plane.

are substituted by decision-making with a very low number of instructions and the numeric evaluation of the switching times gets reduced to a single addition involving the real $\mathbf{v}_{d}$ and imaginary $\mathbf{v}_{q}$ parts of the reference voltage vector. ${ }^{9,10,14}$

\subsection{Determination of the region}

The first step of the method consists of determining the region of the flattened $d-q$ space where the normalized reference voltage vector $\mathbf{v}_{n}^{*}$ is located. The flow chart for determining the region is shown in Fig. 4. 


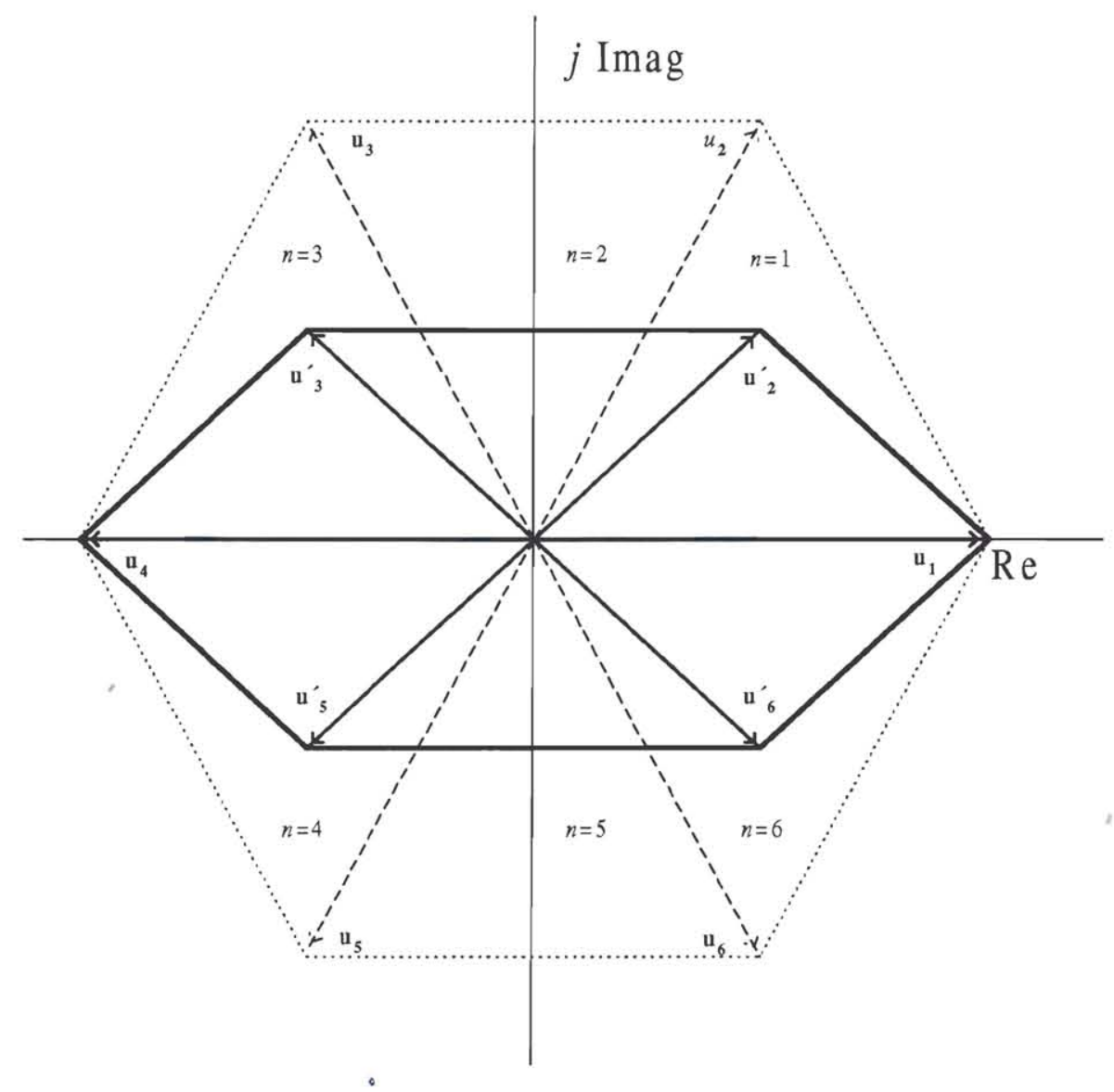

Fig. 3. Switching state vectors before and after the transformation in the complex plane.

\subsection{Calculation of the vertex of a triangle}

The proposed algorithm can be explained while assuming that the reference vector is located in zone 1 . The other two cases are solved in the same way. ${ }^{15}$

The developed algorithm always requires the same computational cost because it is independent of the number of levels of the multilevel converter. This is one of the attractive advantages that this modulation presents if it is compared with most of the algorithms found in the bibliography. ${ }^{4-8}$

For example, the representation of the states of a five-level three-phase inverter in zone 1 is shown in Fig. 5.

$E_{a}, E_{b}, E_{c}$ are the coordinates of one of the vertices of the triangle into the triangle where the reference vector is pointing to. Whatever voltage reference vector located between both lines shown in Fig. 5 fulfils the following expressions:

$$
-v_{d n}+1<v_{q n}<-v_{d n}+2 \Rightarrow 1<v_{q n}+v_{d n}<2 .
$$




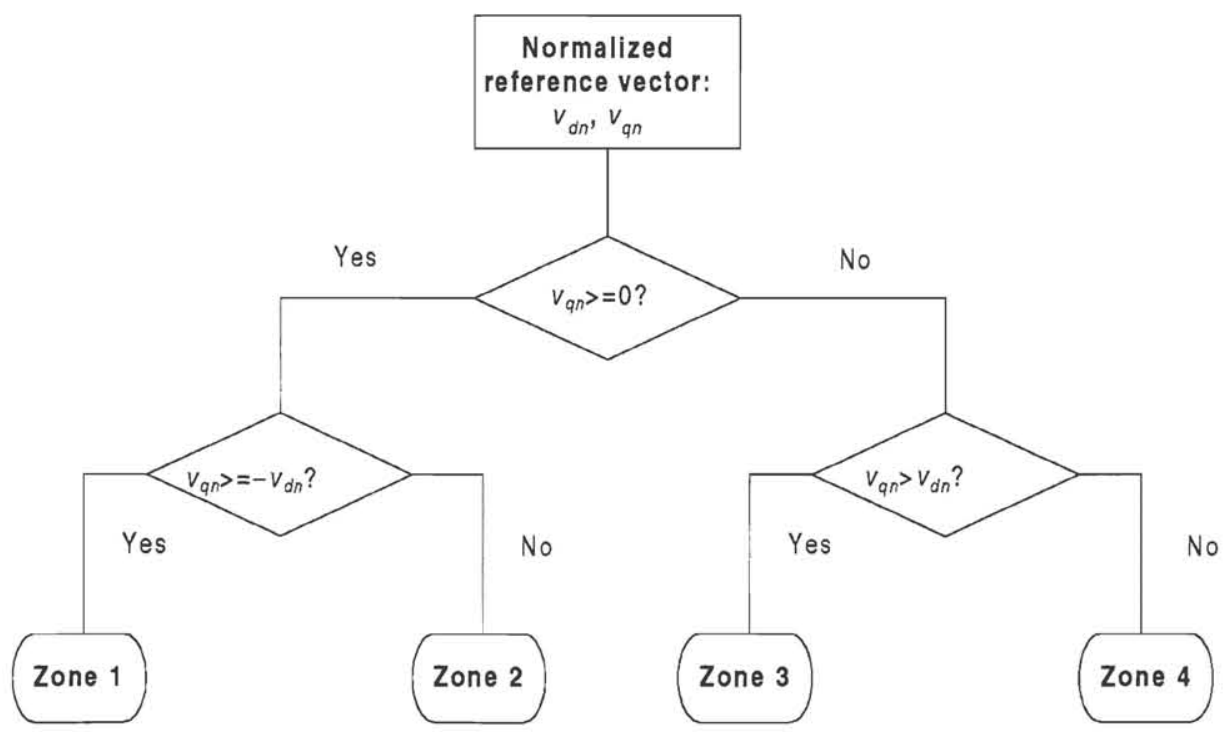

Fig. 4. Flow chart for determining the region in the normalized flattened complex plane.

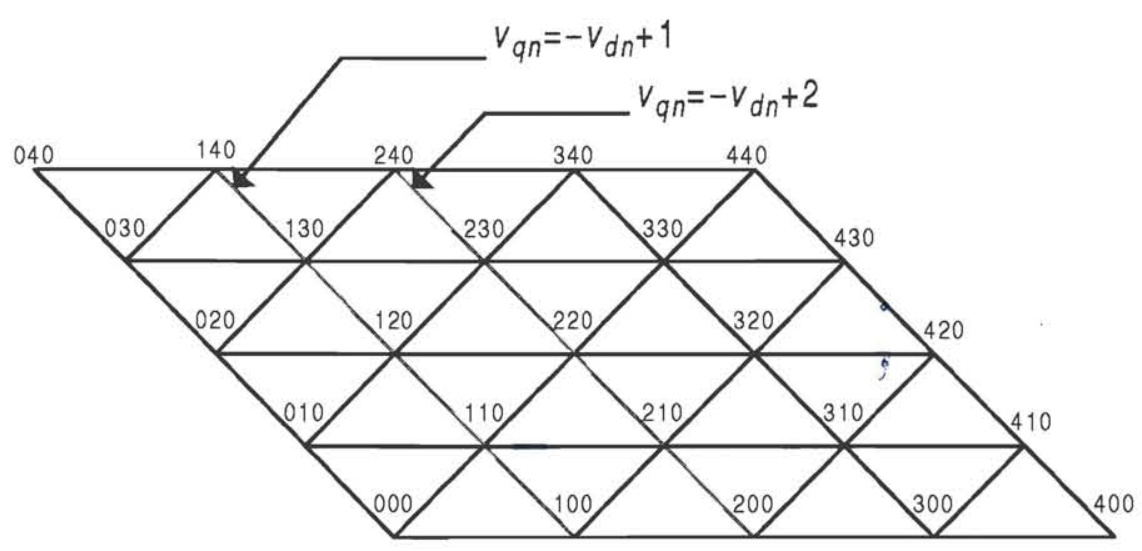

Fig. 5. States of a five-level converter in zone 1.

Every reference vector in this region of the complex plane has one vertex of the triangle where $\mathbf{v}_{n}^{*}$ is found with $E_{a}=1$. In general, this value can be calculated in zone 1 as:

$$
E_{a}=\operatorname{integer}\left(v_{d n}+v_{q n}\right) .
$$

In zone 1 , the $E_{c}$ component is always zero and the $E_{b}$ component is calculated by limiting the region where reference vector is supposed to be found. An example of a limited region in the complex plane by using lines is illustrated in Fig. 6 . 


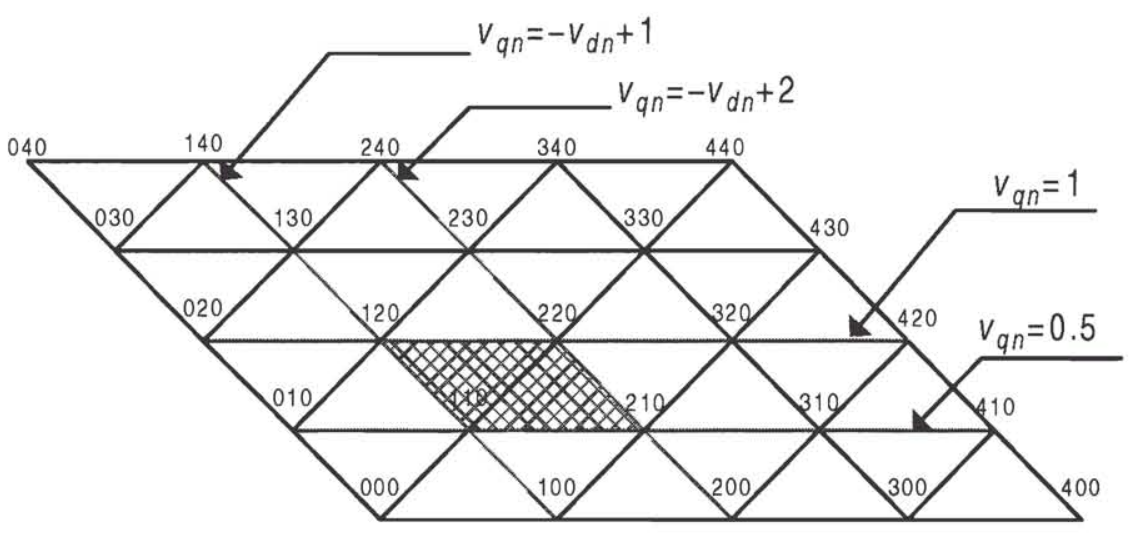

Fig. 6. Limited region in complex plane.

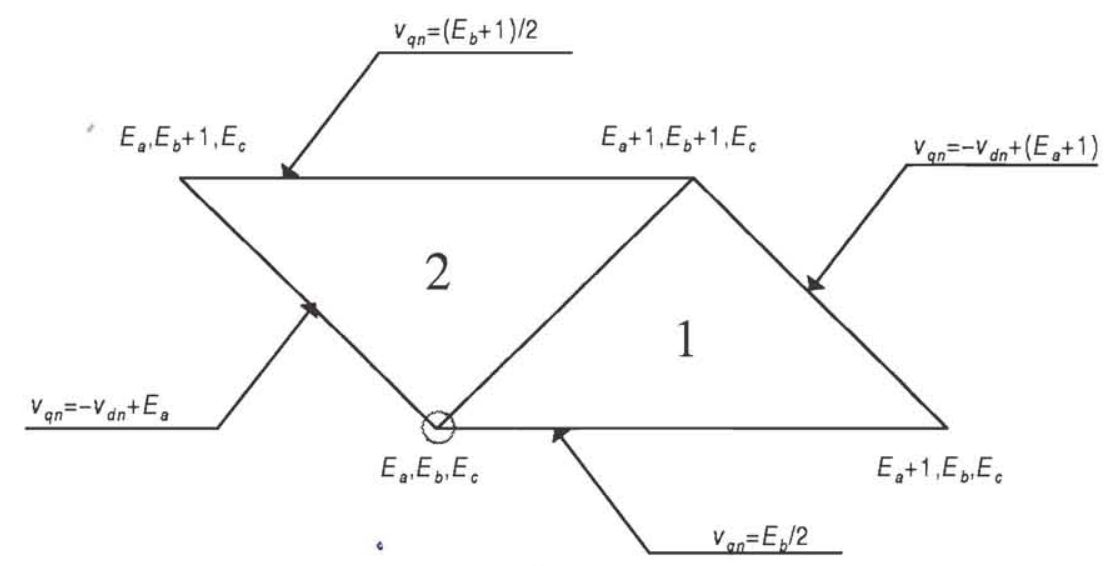

Fig. $\stackrel{7}{7}$. Generic sub-region in zone 1.

Any voltage reference vector in the shown region in Fig. 6 must fulfill the following expression:

$$
0.5<v_{q n}<1 \Rightarrow 1<2^{*} v_{q n}<2,
$$

where $E_{b}$ can be calculated in zone 1 as:

$$
E_{b}=\operatorname{integer}\left(2^{*} v_{q n}\right) .
$$

With this example the calculation of the general expression of the state $E_{a}, E_{b}$, $E_{c}$ of the vertex of a triangle in zone 1 is explained. This method is valid in every sub-region of the zone 1. In Fig. 7, a generic sub-region in zone 1 formed by two triangles with different orientations is presented.

In general, the lower left hand corner vertex of any sub-region in zone 1 can be expressed as: 


$$
\begin{aligned}
& E_{a}=\text { integer }\left(v_{d n}+v_{q n}\right), \\
& E_{b}=\text { integer }\left(2^{*} v_{q n}\right), \\
& E_{c}=\text { " } 0 " .
\end{aligned}
$$

\subsection{Determination of the orientation of the triangle}

Once the lower left hand corner vertex coordinates are known, it is necessary to find out in which of the two triangles shown in Fig. 7 the reference vector located for determining the others states and the switching times is.

If the reference vector is found in the triangle called 1, the geometrical condition in this region is:

$$
v_{q n}<v_{d n}+E_{b}-E_{a} \Rightarrow v_{q n}-v_{d n}<\left(E_{b}-E_{a}\right) .
$$

However, if this geometrical condition is not fulfilled then the reference vector is located in triangle 2.

\subsection{Calculation of the three nearest states to the reference vector}

The three nearest states to the reference vector are obtained when the coordinates $E_{a}, E_{b}, E_{c}$ and the orientation of the triangle are known. In zone 1, they are as follows:

$$
\begin{array}{ll}
\text { Triangle (1) } & \text { Triangle (2) } \\
\text { State 1: " } E_{a}, E_{b}, E_{c} " & \text { State 1: " } E_{a}, E_{b}, E_{c} \text { " } \\
\text { State 2: " } E_{a}+1, E_{b}, E_{c} \text { " } & \text { State 2: " } E_{a}+1, E_{b}+1, E_{c} \text { " } \\
\text { State 3: " } E_{a}+1, E_{b}+1, E_{c} \text { " } & \text { State 3: " } E_{a}, E_{b}+1, E_{c} \text { " }
\end{array}
$$

\subsection{Calculation of the switching times of the active vectors}

The switching times are calculated from the geometrical coordinates of the active vectors. Therefore, the numeric evaluation of the switching times gets reduced to a single addition involving only real and imaginary parts of the reference voltage vector and the coordinates $E_{a}, E_{b}, E_{c}$.

The normalized geometrical components are obtained from the following expression:

$$
\left(\begin{array}{c}
v_{d n}^{E_{a} E_{b} E_{c}} \\
v_{q n}^{E_{a} E_{b} E_{c}}
\end{array}\right)=\left(\begin{array}{ccc}
1 & -\frac{1}{2} & -\frac{1}{2} \\
0 & \frac{1}{2} & -\frac{1}{2}
\end{array}\right)\left(\begin{array}{l}
E_{a} \\
E_{b} \\
E_{c}
\end{array}\right)
$$

where

$$
\left(\begin{array}{c}
v_{d}^{E_{a} E_{b} E_{c}} \\
v_{q}^{E_{a} E_{b} E_{c}}
\end{array}\right)=\left(\begin{array}{ccc}
1 & -\frac{1}{2} & -\frac{1}{2} \\
0 & \frac{\sqrt{3}}{2} & -\frac{\sqrt{3}}{2}
\end{array}\right)\left(\begin{array}{c}
E_{a} \\
E_{b} \\
E_{c}
\end{array}\right) ; \quad\left(\begin{array}{c}
v_{d n}^{E_{a} E_{b} E_{c}} \\
v_{q n}^{E_{a} E_{b} E_{c}}
\end{array}\right)=\left(\begin{array}{c}
v_{d}^{E_{a} E_{b} E_{c}} \\
\frac{1}{\sqrt{3}} v_{q}^{E_{a} E_{b} E_{c}}
\end{array}\right) .
$$




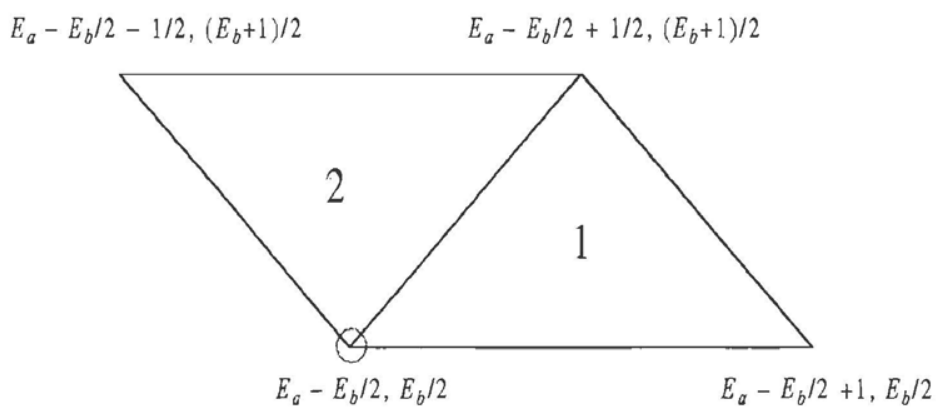

Fig. 8. Geometrical coordinates of a sub-region in zone 1.

Figure 8 shows the geometrical coordinates of the sub-region in zone 1.

The following steps consist of calculating the switching time from the equations:

Triangle 1

$$
\begin{aligned}
d_{1} *\left(E_{a}-\frac{E_{b}}{2}\right)+d_{2} *\left(E_{a}-\frac{E_{b}}{2}+1\right)+d_{3} *\left(E_{a}-\frac{E_{b}}{2}+\frac{1}{2}\right) & =v_{d n}, \\
d_{1} * \frac{E_{b}}{2}+d_{2} * \frac{E_{b}}{2}+d_{3} *\left(\frac{E_{b}+1}{2}\right) & =v_{q n}, \\
d_{1}+d_{2}+d_{3} & =1 .
\end{aligned}
$$

Triangle 2

$$
\begin{aligned}
d_{1} *\left(E_{a}-\frac{E_{b}}{2}\right)+d_{2} *\left(E_{a}-\frac{E_{b}}{2}+\frac{1}{2}\right)+d_{3} *\left(E_{a}-\frac{E_{b}}{2}-\frac{1}{2}\right) & =v_{d n} \\
d_{1} * \frac{E_{b}}{2}+d_{2} * \frac{E_{b}+1}{2}+d_{3} *\left(\frac{E_{b}+1}{2}\right) & =v_{q n}, \\
d_{1}+d_{2}+d_{3} & =1
\end{aligned}
$$

where the switching times $t_{i}=d_{i} T_{m}$, with $i=1,2,3$ and $T_{m}$ is the control cycle. Solving this system of equations for each considered triangle, we obtain the following simple expressions of the duty cycles:

Triangle 1

$$
\begin{aligned}
& d_{1}=1+E_{a}-v_{d n}-v_{q n}, \\
& d_{2}=-E_{a}+E_{b}+v_{d n}-v_{q n}, \\
& d_{3}=-E_{b}+2 v_{q n} .
\end{aligned}
$$


Table 1. Summary of state vectors and the duty cycles.

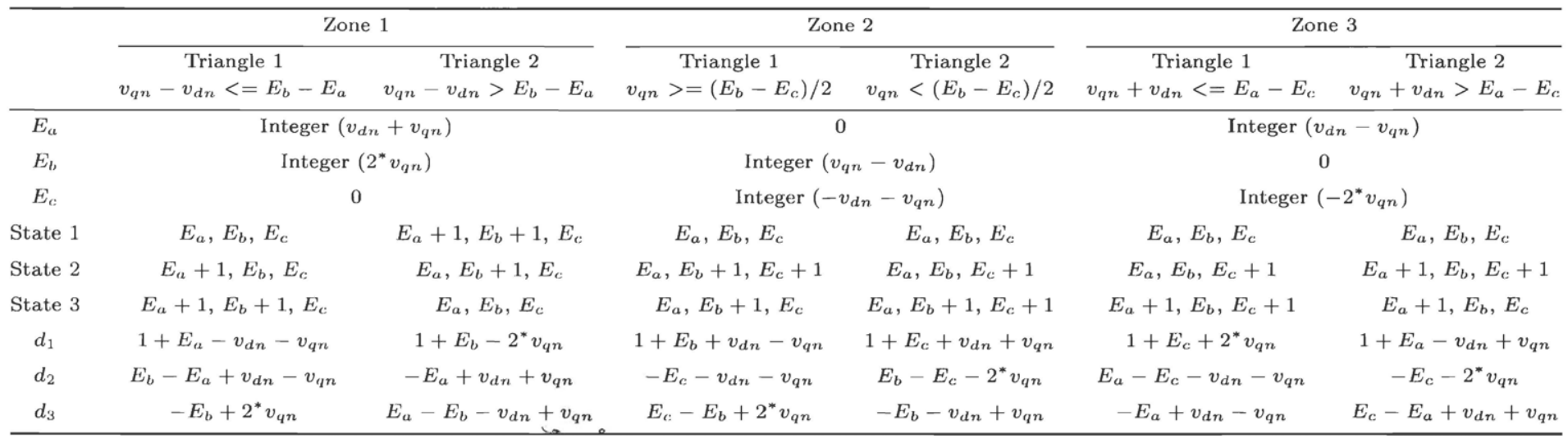


Triangle 2

$$
\begin{aligned}
& d_{1}=1+E_{b}-2 v_{q n}, \\
& d_{2}=-E_{a}+v_{d n}+v_{q n}, \\
& d_{3}=E_{a}-E_{b}-v_{d n}+v_{q n} .
\end{aligned}
$$

\section{Summary of Results}

Table 1 shows summaries of state vectors and the duty cycles in the three regions in the complex plane $d-q$.

\section{New Switching Sequence Control Technique}

For improving the performance of the system using the presented algorithm, a new switching sequence control technique for three-level converters is proposed in this paper for reducing the ripple of the DC-link voltage.

Most switching sequence control techniques balance the DC-link voltage by sampling the conditions in the beginning of each new sequence in a control cycle and provide the switching sequence formed by three state vectors. ${ }^{16}$

The mid-point of the DC-link voltage using a conventional control technique is illustrated in Fig. 9.

However, the voltage ripple can be reduced by choosing each state vector of the sequence according to the corresponding sampled DC-link voltage.

The optimized switching sequences proposed in this work obey the rule showed in Fig. 10 where the five steps of the diagram represent the switching of the power semiconductor devices in each control cycle.

DC-link capacitor voltages and phase currents are sampled in the beginning of each sequence (step 1). Then, the states sequence is calculated. The choice of the sequence affects the mid-point DC-link voltage. Therefore, if the mid-point DC-link voltage is less than 300 volts, all the states of the switching sequence are selected in

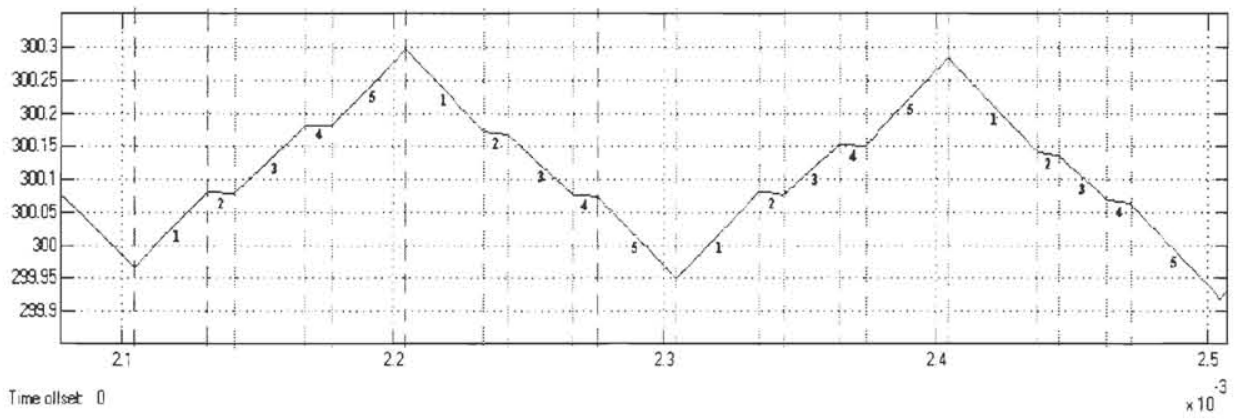

Fig. 9. Evolution of the mid-point DC-link voltage. 


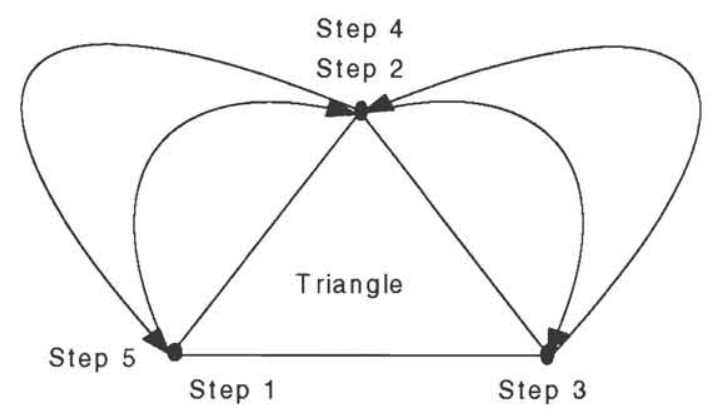

Fig. 10. Rule of switching.

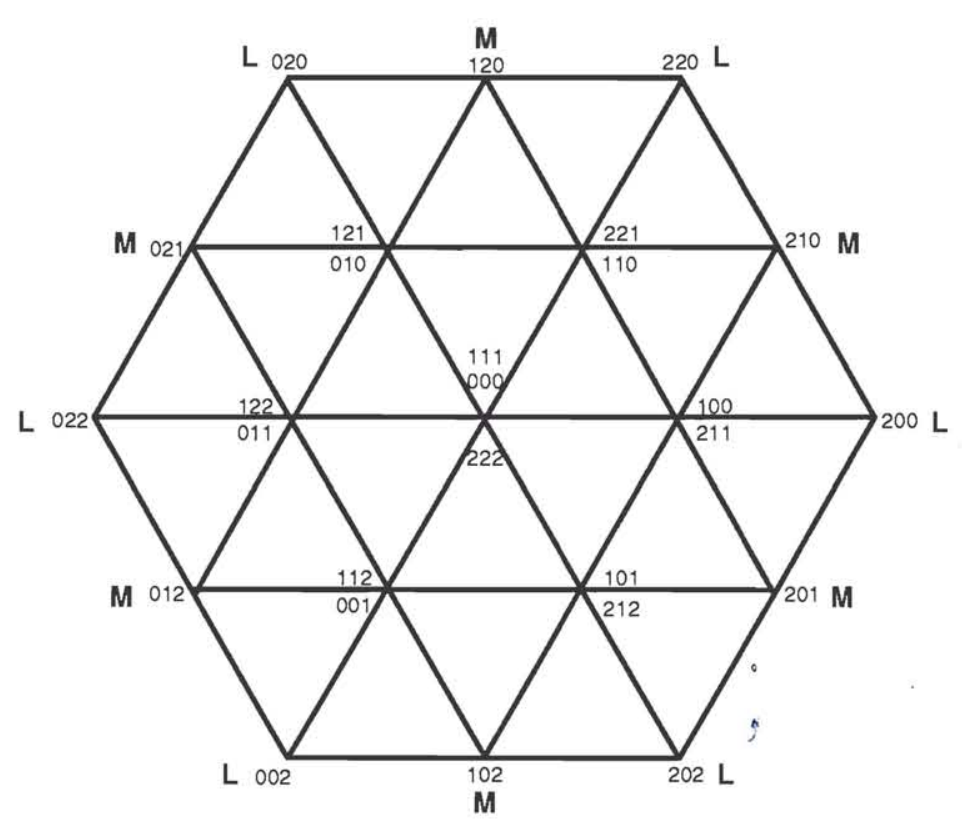

Fig. 11. State space of a three-level inverter.

such a way that the mid-point DC-link voltage is increased. Otherwise, the choice of the switching sequence makes the mid-point DC-link voltage decrease.

In addition, the evolution of the mid-point DC-link voltage depends on the state vector of the sequence. The state vectors are represented as the vertices of the triangle where the reference vector is pointing to. In Fig. 11, the state space of a three-level inverter with the different kinds of vertices is shown.

The action of each vertex on the mid-point DC-link voltage is different. This is shown in Table 2.

In Fig. 9, steps 2 and 4 of the rule show the results of using a vertex with only one possible state. The algorithm must use that vertex and it cannot 
Table 2. Evolution of mid-point DC-link voltage depending on the vertex kind.

\begin{tabular}{ll}
\hline \multicolumn{1}{c}{ Vertex kind } & \multicolumn{1}{c}{ Evolution of the mid-point DC-link voltage } \\
\hline Vertex L & The voltage does not change \\
Vertex M & The voltage cannot be controlled \\
$\begin{array}{l}\text { Vertex with a couple } \\
\text { of states }\end{array}$ & $\begin{array}{l}\text { The voltage is controlled by the algorithm choosing } \\
\text { the best state }\end{array}$ \\
\hline
\end{tabular}

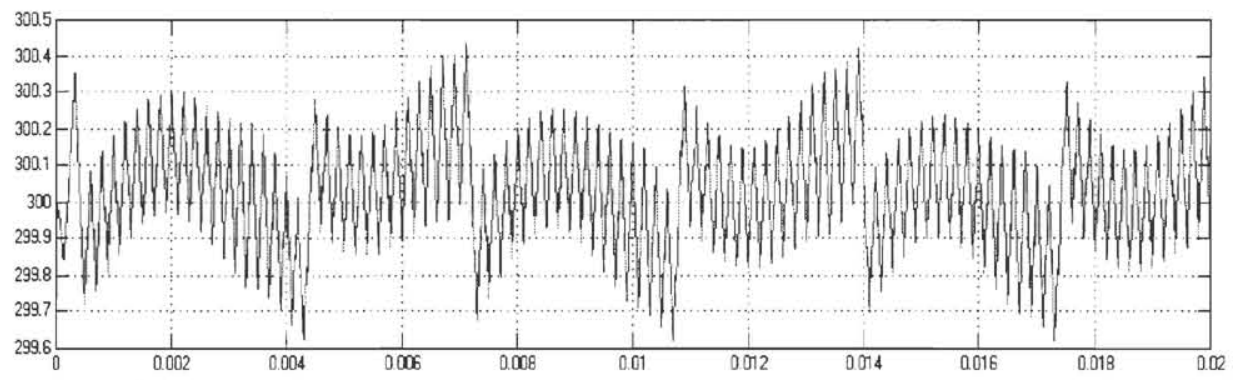

Time offset 0

Fig. 12. Evolution of the mid-point DC-link voltage using conventional algorithms.

choose between different states. In this way, the voltage is not controlled by the algorithm.

In addition, after step 1, the mid-point DC-link voltage is over 300 volts. In this step the conditions have changed and the states have been chosen considering the previous condition. The mid-point DC-link voltage continues increasing from step 2 to step 5. Conventional algorithms sample only once per cycle and the control over the ripple of the mid-point DC-link voltage is less than sampling more times which highly reduces the mid-point DC-link voltage ripple. As Fig. 9 shows, the ripple reaches $0.14 \%$ of the output signal taking 600 volts as DC-link voltage.

The conditions can change while the states remain the same. The algorithm presented in this paper carries out this control taking into account the conditions before each step of the switching sequence. Thus, the used states are chosen independently. If the conditions change, the states to reach a better control will change. This control reduces significantly the ripple of DC-link voltage. The comparison between the evolution of the mid-point DC-link voltage using conventional algorithms and the proposed control algorithm is shown in Figs. 12 and 13:

The ripple reduction reaches $66 \%$ of the signal using this step-by-step control algorithm. A detail of the evolution of the mid-point DC-link voltage using the proposed control is shown in Fig. 14.

These simulation results show an excellent performance of the proposed modulation technique using a new switching sequence control technique for reducing the ripple of the DC-link voltage by approximately $66 \%$. 


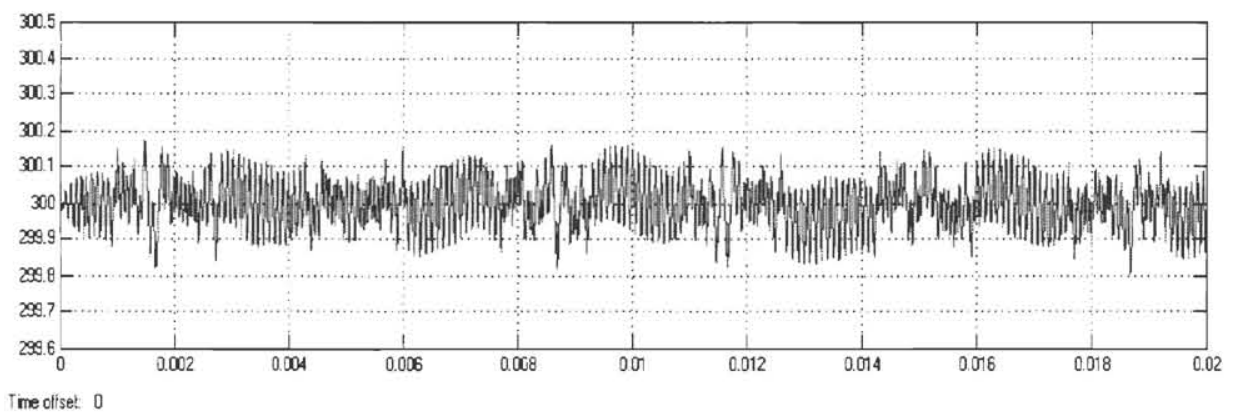

Fig. 13. Evolution of the mid-point DC-link voltage using step-by-step control algorithm.

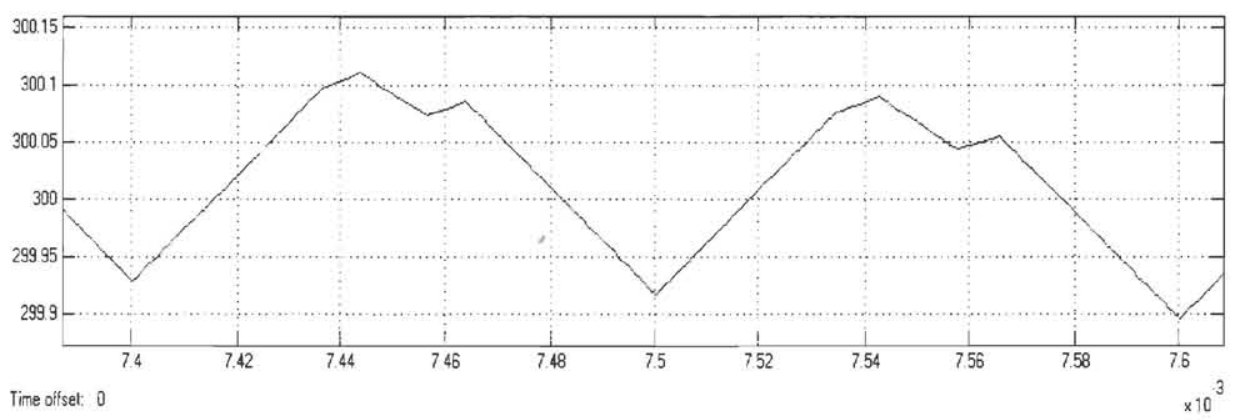

Fig. 14. Detail of the evolution of the mid-point DC-link voltage using step-by-step control.

Solutions using Digital Signal Processors (DSPs) and Field Programmable Gate Arrays (FPGAs) have been studied for implementing this control algorithm.

The simulations shown here have been developed•using Simulink (Matlab). There is a sampler block in the simulated model which samples control signals (phase currents and the mid-point DC-link voltage) with a frequency called $f_{s}$. This frequency is determined by the user. $f_{s}$ must be higher than $f$ (switching frequency). To carry out an efficient step-by-step control algorithm, it must be fulfilled that the frequency ratio, $\mathrm{FR}=f_{s} / f \geq 5$. The presented simulations have been performed with $\mathrm{FR}=5$. However, the $\mathrm{FR}$ factor is limited by the physical implementation of the three-level converter control. If $f=2 \mathrm{KHz}, f_{s}$ would be $10-\mathrm{KHz}$ to carry out the step-by-step control efficiently.

\section{Simulation Results}

\subsection{Simulation results using Matlab}

Figures 16-18 show the simulation results of the modeled system using Simulink (Matlab). A NPC multilevel converter simulation model has been designed by using the corresponding equations in the states space. The semiconductor devices are supposed to be an ideal switch which can be connected to each DC voltage level. 


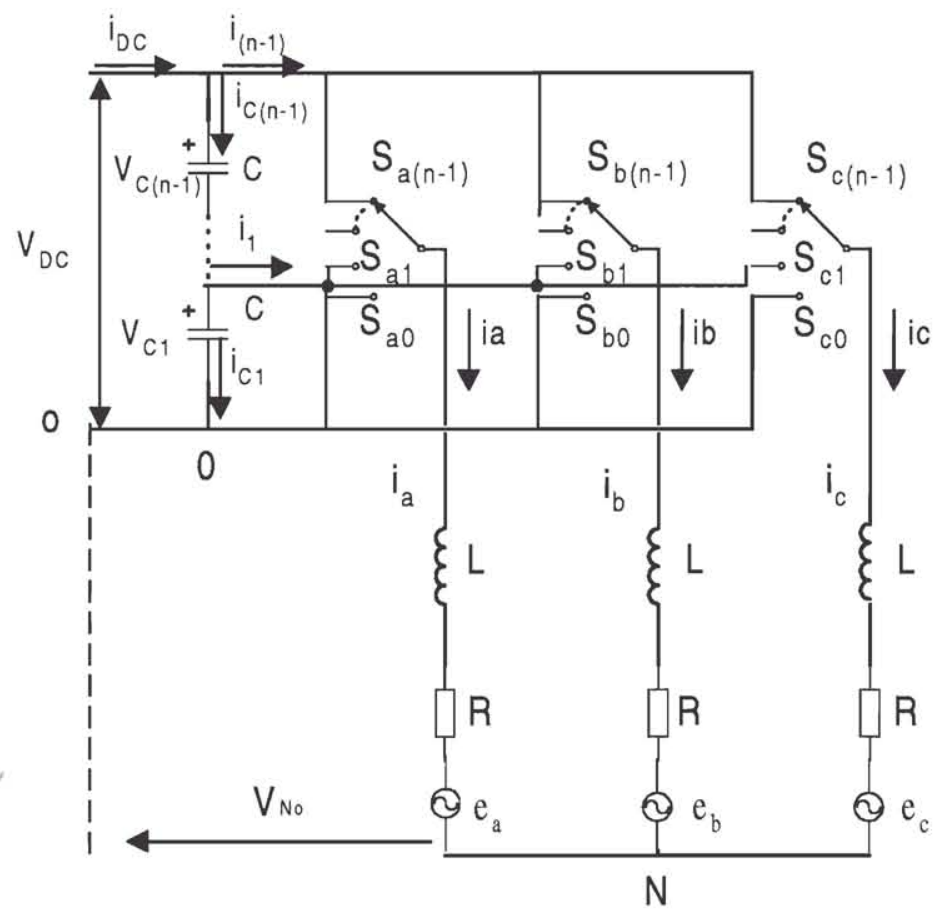

Fig. 15. Multilevel converter simulation model.

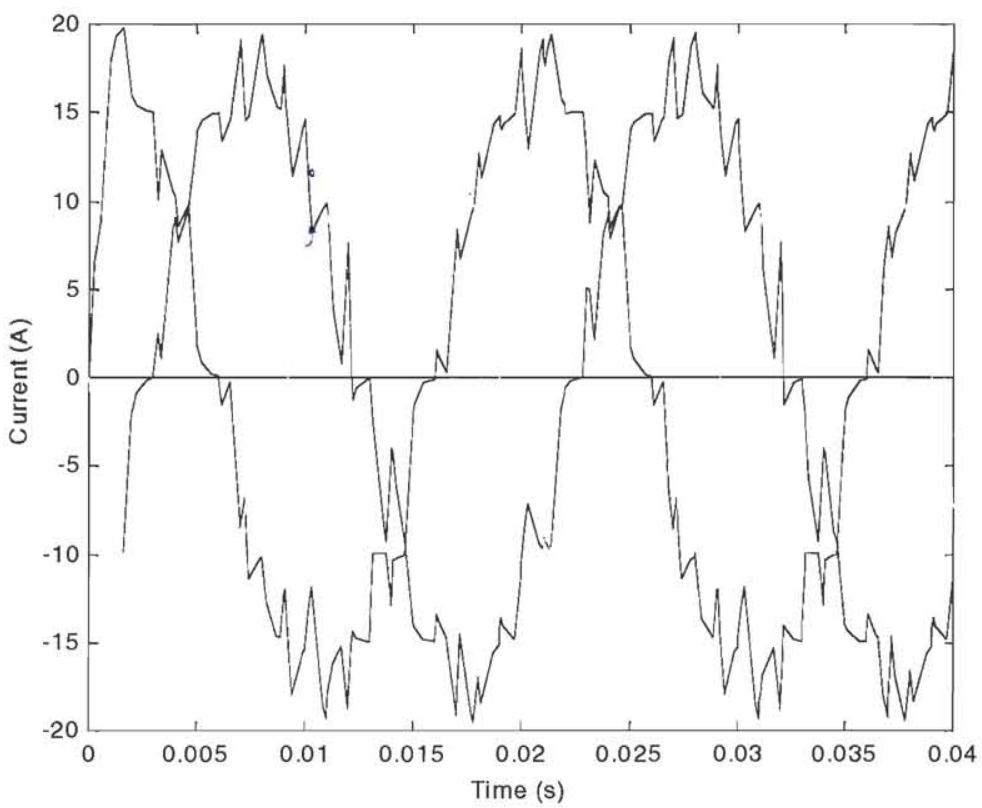

Fig. 16. Phase current of a three-level converter. 


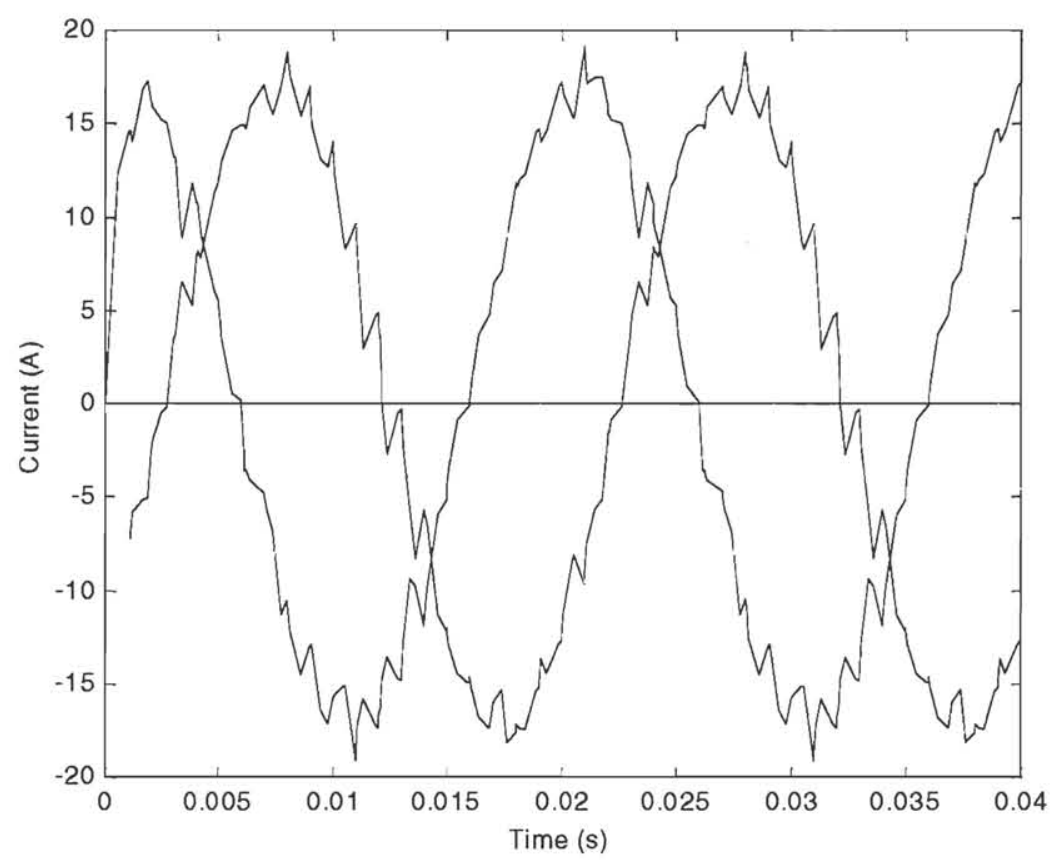

Fig. 17. Phase current of a five-level converter.

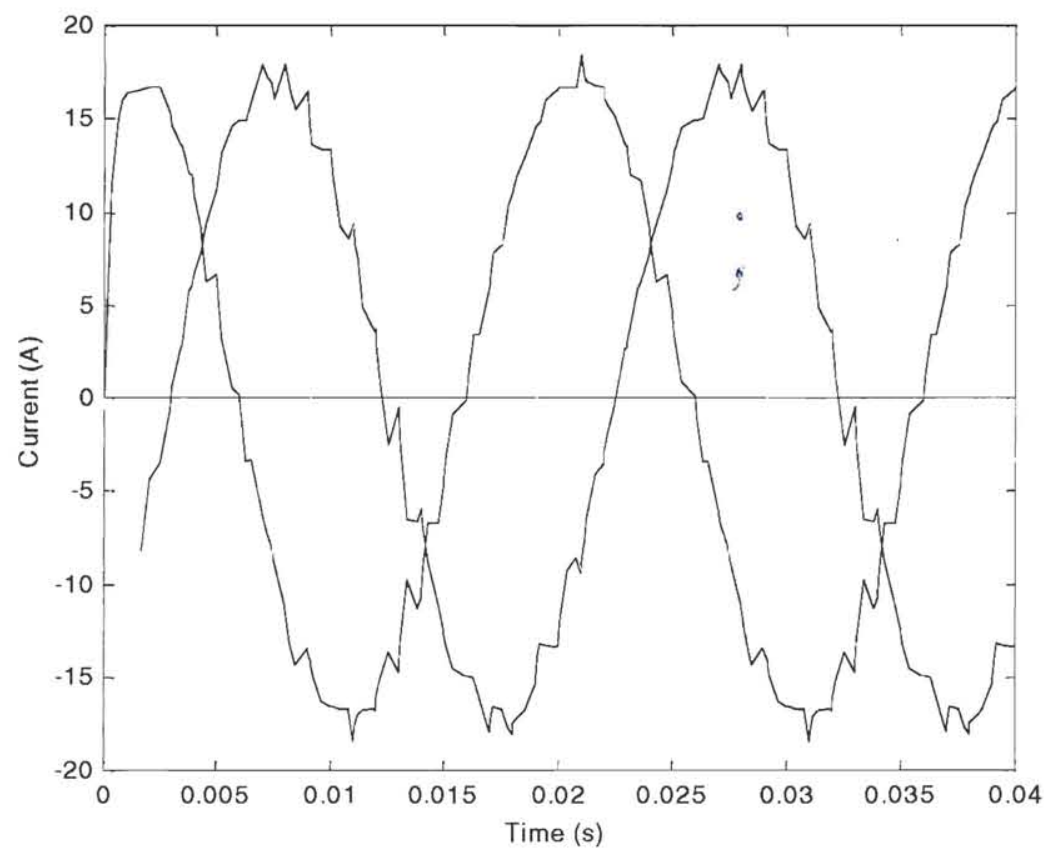

Fig. 18. Phase current of a seven-level converter. 
Table 3. THD results for different frequencies and number of levels.

\begin{tabular}{lrrc}
\hline & $1000 \mathrm{~Hz}$ & $3000 \mathrm{~Hz}$ & $5000 \mathrm{~Hz}$ \\
\hline 3 levels & $12.98 \%$ & $6.30 \%$ & $4.71 \%$ \\
5 levels & $7.52 \%$ & $4.59 \%$ & $3.97 \%$ \\
7 levels & $6.88 \%$ & $3.84 \%$ & $2.83 \%$ \\
\hline
\end{tabular}

The model presented in Fig. 15 can be used in $n$-level multilevel converter. Where each control function $S_{i j}, i=\{a, b, c\}$ and $j=\{0,1,2, \ldots, n-1\}$ take value "1" when the " $i$ " output is connected to " $j$ " voltage level, otherwise take value " 0 ".

The considered operation conditions of the multilevel converter are: switching frequency $f=1000 \mathrm{~Hz}$, DC-link voltage $=600 \mathrm{~V}$ and an $R-L$ load, where $R=20 \Omega$ and $L=5 \mathrm{mH}$. In order to compare the obtained results we are implementing a $50 \mathrm{~kW}$ three-level NPC converter prototype with $900 \mathrm{~V}$ DC-link voltage and $6600 \mu \mathrm{F}$ capacitor.

The effectiveness of the suggested space vector modulation algorithm has been verified by the simulation results using different number of levels of the multilevel converter. The DC-link voltage $V_{D C}$ is supposed to be balanced. This voltage is supplied by an independent source.

Table 3 shows the Total Harmonic Distortion (THD) for switching frequency of 1000,3000 and $5000 \mathrm{~Hz}$ using three-, five- and seven-level converters. These results show an excellent performance of the proposed modulation technique.

\section{Conclusions}

The efficient space vector modulation algorithm presented in this paper is very useful to readily calculate the optimized switching sequence for generating the corresponding reference vector for controlling the switching of multilevel converters. The simulation results show an excellent performance of the proposed modulation technique using a new switching sequence control technique for reducing the ripple of the DC-link voltage by approximately $66 \%$.

The expected results provided by other complicated modulation techniques have been obtained using this algorithm based on simple geometrical considerations. However, the computational burden, the complexity of the algorithm and the number of instructions using the proposed method are drastically reduced compared with other conventional Space Vector Modulation (SVM) and sinusoidal PWM modulation techniques. In addition, the very low computational cost of the proposed method is always the same and it is independent of the number of levels of the converter.

This modulation technique permits an economic and very simple electronic implementation. 


\section{References}

1. A. Nabae, I. Takahashi and H. Akagy, A neutral-point clamped PWM inverter, Proc. IEEE Trans. Ind. Appl. 1A-17 (1981) 518-523.

2. R. Teodorescu, F. Blaabjerg, J. K. Pedersen, E. Cengelci, S. U. Sulistijo, B. O. Woo and P. Enjeti, Multilevel converters - A survey, EPE'99, Lausanne, 1999.

3. J.-S. Lai and F. Z. Peng, Multilevel converters - A new breed of power converters, IEEE Trans. Ind. Appl. 32 (1996) 509-517.

4. L. M. Tolbert and T. G. Habetler, Novel multilevel inverter carrier-based PWM method, Proc. IEEE Trans. Ind. Appl. 35 (1999) 1098-1107.

5. M. Veenstra and A. Rufer, PWM-control of multi-level voltage-source inverters, Power Electronics Specialists Conf. (PESC'2000), Vol. 3 (2000), pp. 1387-1393.

6. R. Rojas, T. Onhishi and T. Suzuki, PWM control method for a four-level inverter, IEE Proc. Electric Power Appl. 142 (1995) 390-396.

7. N. Celanovic and D. Boroyevich, A fast space-vector modulation algorithm for multilevel three-phase converters, Proc. IEEE Trans. Ind. Appl. 37 (2001) 637-641.

8. O. Alonso, L. Marroyo and P. Sanchis, A generalized methodology to calculate switching times and regions in SVPWM modulation of multilevel converters, Proc. 10th European Conf. Power Electronics Appl. (EPE'2001) (2001).

9. M. M. Prats, J. M. Carrasco and L. G. Franquelo, Effective modulation algorithm for three-level converters, Proc. PCIM '2002, Nuremberg, Germany (2002).

10. M. M. Prats, J. M. Carrasco and L. G. Franquelo, Effective algorithm for multilevel converter with very low computational cost, Proc. IEE Electron. Lett. 38 (2002) 1398 1400 .

11. R. Teodorescu, F. Blaabjerg, J. K. Pedersen, E. Cengelci and P. N. Enjeti, Multilevel inverter by cascading industrial VSI, Proc. IEEE Trans. Ind. Electron. 49 (2002) $832-838$.

12. B. M. Song, S. Gurol, C. Y. Jeong, D. W. Yoo and J. S. Lai, A soft-switching highvoltage active power filter with flying capacitors for urban maglev system applications, Proc. Ind. Appl. Conf. (IAS '01) (2001), pp. 1461-1469.

13. N. Celanovic and D. Boroyevich, A comprehensive study of neutral-point voltage balancing problem in three-level neutral-point-clamped voltage source PWM inverters, Proc. IEEE Trans. Power Electron. 15 (2000) 242-249.

14. M. M. Prats, J. M. Carrasco and L. G. Franquelo, Effect r̂ve space-vector modulation algorithm for multilevel converters, Proc. 28th Annual Conf. IEEE Ind. Electronics Society (IECON'02), Sevilla, España (2002).

15. M. M. Prats, R. Portillo, J. M. Carrasco and L. G. Franquelo, New fast pace-vector modulation for multilevel converters based on geometrical considerations, Proc. 28th Annual Conf. IEEE Ind. Electronics Society (IECON'02), Sevilla, España (2002).

16. B. P. McGrath, D. G. Holmes and T. A. Lipo, Optimised space vector switching sequences for multilevel inverters, Applied Power Electronics Conf. Exposition (APEC 2001) 16th Annual IEEE, Vol. 2, 2001. 